Cahiers de Narratologie

\title{
Révéroni Saint-Cyr et le décor gothique. De la topique spatiale à la fonction symbolique
}

\section{Maria Concepción Pérez}

\section{(2) OpenEdition}

1 Journals

Édition électronique

URL : http://journals.openedition.org/narratologie/11633

DOI : 10.4000/narratologie.11633

ISSN : 1765-307X

Éditeur

LIRCES

\section{Édition imprimée}

Date de publication : 1 décembre 1997

Pagination : $311-321$

ISBN : 291089746X

ISSN : 0993-8516

\section{Référence électronique}

Maria Concepción Pérez, « Révéroni Saint-Cyr et le décor gothique. De la topique spatiale à la fonction symbolique », Cahiers de Narratologie [En ligne], 8 | 1997, mis en ligne le 15 décembre 2020, consulté le 25 février 2021. URL : http://journals.openedition.org/narratologie/11633 ; DOI : https://doi.org/ 10.4000/narratologie.11633

Ce document a été généré automatiquement le 25 février 2021.

Article L.111-1 du Code de la propriété intellectuelle. 


\title{
Révéroni Saint-Cyr et le décor gothique. De la topique spatiale à la fonction symbolique
}

\author{
Maria Concepción Pérez
}

1 Brillant dans l'univers imaginaire de Révéroni Saint-Cyr, le roman par lequel il inaugure sa production littéraire, Pauliska ou la perversité moderne, mémoires récents d'une polonaise $(1798)^{1}$, constitue l'ouvrage le plus représentatif de ce genre - le roman noir - que les Anglais appellent gothique, et qui se développe en France dans la frontière grouillante entre le XVIII ${ }^{e}$ et XIX ${ }^{e}$ siècle. Du point de vue de l'analyse de l'espace, ce roman perdu dans les bibliothèques jusqu'à une date assez récente, nous offre un champ de réflexion intéressant, malgré l'impression trompeuse qu'une première lecture pourrait produire, encadré qu'il est dans la topique qui caractérise le genre et l'époque. En effet, fruit de ce foisonnement romanesque caractéristique du XVIII siècle, qui génère une transposition de formes où les clichés s'empruntent et se combinent, le roman noir comporte une topographie spécifique, peuplée de châteaux à tours élevées et souterrains profonds, de ruines et couvents aux entrailles tortueuses, labyrinthiques, où le vertueux se voit toujours confronté au méchant, au pervers, où les limites sont transgressées dans les frontières entre la réalité et le rêve, le tangible et l'imaginaire.

2 Dans ce roman, l'espace apparaît dessiné à grands traits, aux fortes couleurs, suivant une économie descriptive qui produit l'effet d'un expressionnisme presque enfantin. Or, il s'agit d'une plasticité essentiellement sémiologique, qui présente un caractère important en ce qui concerne la structure narrative et interprétative par rapport à l'Histoire, remplissant des fonctions diverses selon que les espaces soient parodiques, grotesques, pseudo-utopiques ${ }^{2}$ ou métonymiques. Ma prétention dans cet article consistera alors à ouvrir un trajet symbolique qui, de la topique du décor "gothique ", plus ou moins ornementale, conduit à la métonymie et à la métaphore de l'Histoire.

3 Pauliska est le récit d'une expulsion, la longue fuite de l'héroïne lors de l'invasion de la Pologne par les troupes de Catherine II de Russie. Au premier abord, on dirait que 
l'Histoire ne constitue qu'une toile de fond, tandis que, outre le fait que les aventures vécues par les protagonistes offrent déjà une satire des valeurs perverties des Lumières, l'Histoire devient prégnance thématique, comme substance profonde qui émerge et accorde sa coloration au récit.

Les espaces parodiques présentent un caractère ambivalent, témoignant en même temps d'une nostalgie et d'un échec historiques. Il s'agit essentiellement d'espaces sociaux, notamment de celui de la cour aristocratique, mirage doré, appel joyeux, nostalgie des mœurs que l'on voudrait conserver, d'une époque de gloire et puissance féodales. Mais l'Histoire a sonné le glas d'un âge révolu, l'ironie veut que ce soit en paysanne que Pauliska reçoive les hommages de la société ${ }^{3}$, et le malheur se déchaîne après l'éclat apparent de bonheur. Notons que l'ironie peut aller jusqu'à la configuration d'un véritable espace grotesque, comme c'est le cas de l'armée de réfugiés composée par le Corps franc de Giulai ou d'une manière bien mieux plastique le club de Tornik, où ses membres - des chiens habillés - se déchirent entre eux pour des débris de nourriture sous la devise système agraire, bonheur commun.

Mais si les espaces parodiques présentent un caractère ambivalent, ceux qu'on pourrait qualifier de pseudo-utopiques le sont à leur tour. Ainsi, idéalité et déception coexistent dans l'expression apocalyptique d'un monde qui éclate chaotique en marquant la fin des temps. Si Pauliska se reconnaît «jouet d'une révolution dont personne n'a pu calculer les suites » (page 31), ce phénomène ne se circonscrit pas à son pays, car «l'explosion devait être universelle, la vertu ou les torts, les bienfaits ou les griefs, devaient être confondus et une race entière sacrifiée » (page 32). L'image de l'explosion ne relève pas de la pure rhétorique, ayant une efficacité du point de vue événementiel et interprétatif.

6 D'après cette ambivalence que je viens de signaler, l'espace pseudo-utopique fait son apparition après une première expulsion de l'espace historique réel ; par conséquent il comportera la nostalgie du retour, en même temps qu'il abrite les membres d'une régénération future.

7 Il en est ainsi de la microsociété qui habite le refuge de glace, et dont le noyau n'est qu'une transposition de celle qui constitue l'entourage de Pauliska au début du roman. Le hasard marque l'arrivée dans une nouvelle et magique dimension, figurée comme une chute dans l'abîme :

J'ignore ce qui se passa ; mais qu'on juge de ma surprise, lorsque je me crus à mon réveil dans un palais de cristal, aux clartés de mille flambeaux, déposée mollement sur une estrade, environnée d'un peuple soumis ! (page 71)

Remarquons que pour Pauliska l'ordre idéal apparaît d'abord comme un retour au passé, comme un maintien du statu quo. La réalité pourtant manifeste rapidement son caractère incontestable :

Malheureuse ! ce palais n'était qu'une grotte de glace, humide, éclairée d'une lampe funèbre, mon trône était un lit de neige qui m'avait sauvée dans ma chute; et ce peuple de sujets, une société de malheureux transfuges comme moi, sans espoir, sans pain, sans secours, (ibid.)

9 Le palais flamboyant de cristal devient grotte lugubre et humide où se rassemblent les réfugiés polonais, d'après une dynamique dans le mouvement d'appréhension spatiale qui fonctionne comme une constante et où un premier effet d'irréalité qui transforme l'espace en lieu onirique, laisse brusquement la place à la réalité la plus criante. Or après ce stade intermédiaire, le micro-cosmos spatial prend une nouvelle définition, où 
l'ambivalence se manifeste en termes antithétiques: ainsi, si d'une part les réfugiés ne feraient qu'attendre la fin du monde, la totale destruction d'une apocalypse de feu et de sang qui n'épargnerait même pas "un seul juste", ce noyau rassemble une société vertueuse où la solidarité constitue la base solide de régénération, où chacun doit être utile à la communauté, en y apportant le fruit de son travail ou de son expérience, où, malgré le caractère patriarcal de la structure, les femmes configurent le pilier le plus solide.

Un deuxième modèle de rêverie utopique viendrait constituer par la société campagnarde groupée autour du modeste hameau de Jeanna, "sous un rocher à pic, dominé par deux sapins» (page 80) aux échos rousseauniens. La vertu et l'harmonie ordonnent une vie paisible à l'intérieur de laquelle à nouveau le travail devient la valeur basique d'appui. Nous constatons également à nouveau la présence de l'élément non seulement réaliste, mais issu de la réalité elle-même, car la famille de Jeanna elle aussi est conformée par des exilés (autrichiens) ayant perdu leur condition aisée : l'Histoire a rendu le travail la seule valeur de survivance et autonomie possible.

D'autre part, la considération sociale ne vient pas donnée par l'origine de l'individu, mais par son éducation, ce grand rêve des Lumières qui croient par cette voie à la perfectibilité humaine. Ainsi, parmi le voisinage, Jeanna jouit d'une spéciale considération «que j'attribuai d'abord à son origine - avoue Pauliska - mais que je reconnus bientôt être le fruit de son éducation soignée » (page 83). Quant à l'amour, celui-ci est conçu sur les bases de l'égalité, de telle façon que Jeanna "a voulu se donner un ami et non un maître » (ibid.). Mais - hélas ! - le bonheur ne peut pas être complet, car l'isolement des individus par rapport à l'Histoire est impossible, le déchaînement révolutionnaire trempe partout la peau de l'Europe et il n'y a pas d'espace sûr pour les amants : utopie et réalité finissent bien par se rejoindre et s' entr' équilibrer.

De tous les emplois de l'espace, sans doute c'est à travers la fonction métonymique qu'il acquiert ses meilleures résonances, la métonymie pouvant aller d'une fonction purement topique jusqu'à des fonctions plus complexes. Ainsi, conduite par le hasard de l'amour dans le lac de Falsback, suivant un emploi topique du cliché romantique, le paysage devient expression de Pauliska :

Je m'impatientais, et sortant à pied, je louai une voiture, et me fis conduire sur le chemin du lac de Falsback. Comme mon cœur battit avec violence, quand je découvris sur les bords du lac, le village et le quartier des réfugiés polonais! Le soleil se couchait alors et dorait toute la rive opposée d'une teinte rouge et brillante, tandis que le bord sauvage où je me trouvais conservait cette teinte lugubre des forêts et des rochers dans l'ombre. En un mot, c'était devant moi l'éclat trompeur de la gloire ; ici, la sombre obscurité de l'amour inquiet. (page 45)

13 Ailleurs les images peuvent prendre une teinte plus romanesque, comme dans l'épisode de la rencontre des amants désormais séparés par l'obstacle insurmontable du mariage d'Ernest : leur ombre reflétée du haut de la tour sur les eaux éclairées par la lune et que Julie, jalouse, poignarde, devient le substitut de leur corps et le symbole d'une union coupable.

14 Mais si dans ces cas la dimension symbolique est ramenée à un point zéro, elle peut bien acquérir son sens propre, suivant différents degrés de signification. Alors les noires entrailles de la terre ne sont autre chose que l'exploration des abîmes de l'être poussé par l'Histoire. Alors l'emploi des couleurs contrastées cesse d'être un élément du décor pour devenir charge signifiante : la blancheur immaculée de la neige cache la 
noire obscurité qui abrite les réfugiés polonais dans les entrailles du mont Stolberg, effaçant toute trace, tout signe, faisant de l'espace une table rase d'où la mémoire a disparu. Mais cette blanche surface sans mémoire cache un monde grouillant de survivants, derniers témoins d'un monde qui va se détruire. Arrivés à ce point, lorsque la rêverie de l'Histoire prend des teintes apocalyptiques la métonymie spatiale opère une corporéisation de l'espace. Ainsi, lorsque le vieil évêque prophétise l'irrémédiable apocalypse, tous les corps deviennent un seul et même « faisceau » de chair :

«Nos malheurs seront grands, s'écriait-il d'un ton prophétique; que celui d'entre vous qui a cru n'éprouver qu'une tribulation passagère, qui a pensé que la raison pût triompher d'une soldatesque effrénée, se détrompe. La vertu sera comprimée et vaincue, tous les peuples égarés vont s'entre-détruire, des vapeurs de sang s'élèveront jusqu'aux cieux, le soleil en sera obscurci, la terre frémira des forfaits des mortels, et dans son tremblement ouvrira leur tombe, sur laquelle la voûte des cieux viendra s'abattre et sceller leur réprobation éternelle. Malheur ! malheur aux peuples! il n'est plus un seul juste, digne du regard du Très-Haut !...»

A ces mots terribles, la lampe tomba avec le glaçon auquel elle était suspendue. Les ténèbres, les sanglots de tant d'infortunés errant dans l'obscurité, se cherchant, se heurtant, ne formant qu'un faisceau de leurs bras tremblants, ce désordre horrible, fruit de cette prophétie et de nos craintes, me laissèrent un souvenir ineffaçable. (page 73)

15 Mais c'est la terre elle-même qui est faite de corps, des morts entassés qui ont cru au mirage de la liberté, ainsi que fait constater le père de Jeanna :

« Avez-vous vu, dit-il, les champs de Zurich?

Ils sont blanchis par les ossements des impériaux ; avez-vous vu les retranchements $\mathrm{du}$ lac ? Ils sont formés de leurs crânes entassés! Toutes ces têtes aussi ont cru à la victoire et tombent en poussière depuis des siècles dans les mêmes champs qu'elles ont cru reconquérir. Voilà le sort destiné aux infortunés Polonais. » (p. 82)

La liberté n'est autre chose que le "jouet du politique", de la même façon que « l'électricité l'est du physicien » (page 82), ravageant la terre, car finalement elle ne fait qu'apporter « les commotions, les orages et la destruction » (ibid.). A la lumière de cette comparaison le rôle joué par Salviati, magicien de l'électricité, prend une signification plus profonde que celle purement anecdotique, devenant image du politique, métaphore de l'Histoire.

L'espace devenu chair peut balancer sa polarité aussi bien sur l'Histoire que sur le moi. L'image cosmique peut métaphoriser l'Histoire, comme dans les exemples que nous venons de considérer, mais le moi peut être aussi l'image du Cosmos, et donc de la nature. Ainsi, le visage du baron d'Olnitz, où « [ces] traits altérés par une imagination de feu, ce front calciné par des idées volcaniques ont devancé par leurs rides les impressions de l'âge » (page 187) devient l'expression même de l'énergie cosmique.

A l'inverse, l'image du volcan devient métaphore de l'Histoire, de la destruction à laquelle l'Europe révolutionnée est vouée. Ainsi, au siège de Kostheim, la bataille finit par prendre une dimension cosmique et apocalyptique :

Mais tandis que nous franchissions les fossés et pénétrions de toutes parts dans l'ouvrage, un fracas épouvantable surprend tout à coup, bouleverse, enlève dans les airs une partie des assaillants. Une atmosphère de soufre nous suffoque, la terre ébranlée, entrouverte, vomit ses entrailles de feu jusques aux cieux et soudain rappelle et engloutit en son sein mille infortunés qu'elle y avait lancés. Une mine effroyable emporta la moitié du Corps de Giulai. (page 134)

19 Remarquons comment à nouveau, lorsqu'il se produit cette dérive universelle, le corps thématise une image collective, et de la même façon que dans le refuge souterrain tous 
les bras ne faisaient qu'un seul faisceau de bras, le corps de l'armée perd la banalité de cette dénomination pour devenir véritable chair de chairs.

Dans cette dérive vers l'universalité les espaces deviennent des images les uns des autres, dans un rapport d'équivalence. Mais, en plus, passé et présent se confondent, de telle façon que les ruines du passé deviennent les témoins présageant de la destruction présente. Ainsi, Rome est vue par Pauliska comme l'image de la Pologne :

J'arrivai à Rome à la fin de juillet. Quelle émotion j'éprouvai à la vue des vestiges de cette antique reine de l'univers! Quelle décadence! Quel tableau du néant de la gloire et des passions des hommes! Je songeai aussi aux désastres de ma patrie, et c'est dans ces tristes réflexions que je descendis à un hôtel modeste, sur la place du Cirque. (Page 179)

21 En ce qui concerne la rêverie de la destruction, l'image de l'explosion remplit une fonction importante. Faisant partie de sa sémiologie, la métonymie du rouge joue un rôle particulièrement actif, et constitue une constante tout au long du roman, en permettant d'établir des parallélismes entre des personnages et des situations.

Dans ce roman aux forts contrastes la froideur de la glace coexiste avec le feu le plus ardent. A cet égard, un exemple achevé vient constituer par la maison des fauxmonnayeurs ensevelie dans les eaux du Danube, possédant, au lieu de fenêtres, des houblots que couvrent les eaux du fleuve. L'intérieur pourtant est entièrement construit sur la métonymie du rouge, qui atteint aussi l'élément humain, de telle façon que Talbot, le chef des faux-monnayeurs, possède des sourcils et des cheveux rouges qui, lorsqu'ils se hérissent, semblent « se poudrer de sang » (page 97).

La maison aquatique devient, à partir de la métonymie du rouge, un espace de sang et de feu. Ainsi, le lieu de travail de Pauliska est présidé par un « secrétaire noir, marqué de taches rouges en bois incrusté, et qui jouaient le sang à faire horreur» (page 92). Mais, en plus, ce spectacle est complété par la corporéisation de l'espace, qui se construit, comme l'espace historique, sur le substrat des corps sacrifiés. Pauliska avoue: "J'ouvre ce secrétaire, quel spectacle, grand Dieu! l'encre dans un crâne d'ivoire, les chandeliers... des faisceaux d'ossements, portant une petite bougie lugubre, et les tablettes couvertes d'une série de poisons en fioles et étiquetés!» (ibid.). Le lieu se génère donc sur la mort, mais en plus il est générateur de mort, car l'ancien couvent des cordeliers fonctionne comme espace nucléaire qui distribue la mort en Europe, en contrefaisant des sentences. La contrefaçon (sentences, assignats, etc.), activité quotidienne des faux-monnayeurs, rayonnant à partir d'un espace historique caractéristique du roman noir - suppose une perversion de l'Histoire.

Le rouge se présente d'autre part comme métonymie du feu : lorsque la sentence contre Pauliska et Durand va être prononcée, dans la salle du conseil, où la table est couverte d'un tapis rouge, "un vase rouge rempli de billets, des flambeaux étincelants, tout donnait à cette assemblée une teinte de feu, un air lugubre et incendiaire » (page 97).

Or, dépassant le pur niveau événementiel, cet incendie atteint l'Europe entière, car il s'agit d'un espace nucléaire, "foyer du machiavélisme anglais », dont le but est «la destruction totale [de l'Europe] et non le triomphe d'un parti que ce gouvernement désire » (page 91).

26 A cet égard, Rome remplit elle aussi une fonction nucléaire. Cet espace qui, par son substrat historique, métonymise le monde entier, est le lieu où réapparaissent, en s'y ajoutant de nouveaux, les persécuteurs de Pauliska. Rome, image fréquente au tournant des lumières, est vue à travers la métonymie du spectacle et de la théâtralité, du jeu 
entre apparence et réalité. Et à Rome, l'espace privilégié où se concentre l'action est le château séculaire de Saint-Ange, siège de justice. A son tour, le château est métonymisé dans la présence du souterrain, lieu typique du roman gothique. Là, l'espace corporéisé dans la métaphore de la chevelure se transmue en pleurs des prisonniers enfermés :

Je ne m'arrêterai pas à décrire les antres sombres, les ponts voûtés en fer, sous lesquels les bras du Tibre comprimés s'éloignent en bouillonnant; les cavernes couvertes d'une mousse humide, chevelure hideuse de rochers éternels [...]. quelques lampes rares projetant des ombres immenses sous ces voûtes; des sbires qui n'ayant vu le jour depuis vingt ans, ont la pâleur des spectres; des gouttes d'eau qui coulant des murs des cachots sur nos têtes, semblaient être l'infiltration des pleurs des malheureux prisonniers, sont les seuls tableaux dont le souvenir me reste, et dont l'impression horrible est ineffaçable. (page 196)

Le souterrain, où la lumière, au lieu d'éclairer, ajoute à l'obscurité, décrit un trajet labyrinthique, composé «de longs et mystérieux détours », coupés de temps en temps par des carrefours, devenant l'expression de l'égarement de l'être dans le carrefour de l'Histoire. Or, signe de l'impossible isolement de l'individu par rapport à celle-ci, la prison impénétrable est perméable à la réalité extérieure, et les événements historiques - que l'on cache avec soin - arrivent jusqu'aux prisonniers.

Du point de vue de la fonction métonymique le souterrain devient espace de feu lui aussi, corporéisé dans les soldats "flamboyants » qui configurent une image d'enfer, ou, mieux encore, dans la disparition de Zéphirine, « cet être de feu, au physique et au moral », qui meurt consumée par les flammes.

Mais à l'intérieur de la prison, la métonymie sonore est particulièrement significative.

A travers elle, le jugement des prisonniers devient le reflet du jugement dernier :

Enfin l'instant terrible arriva: celui du jugement général. Je ne crois pas que la trompette de l'Ange redouté, sonnant dans le dernier jour des mortels produise un effet plus effrayant, que le bruit des gonds et ferrements de la grande porte des juges du tribunal qui s'ouvrit pour nous. (page 206)

La métonymie sonore et celle du feu finissent par se conjuguer dans l'image de l'explosion finale qui amène la délivrance des prisonniers. C'est le moment de l'Apocalypse: après le bruit de "l'explosion épouvantable ", «les voûtes fendues, brisées à l'extrémité du souterrain, laissent arriver un jour rouge à travers une fumée épaisse » (pages 207-208), et l'explosion qui a lieu sur le plan événementiel renvoie à la métaphore de l'explosion universelle où griefs et vertus se confondent dans une Europe révolutionnée. Malgré le long oubli que ce roman a subi, ainsi que son auteur, aussi bien de la part des lecteurs que de la critique, il pourrait être pris pour emblème de cette époque, car Pauliska ou la perversité moderne se pose en témoignage et présence critique face à l'Histoire, une Histoire qui devient machinerie folle par suite du déchaînement révolutionnaire qu'est devenue l'Europe. A cet égard l'image de la machine - cloche pneumatique où Pauliska est enfermée, ou encore l'appareil électrostatique où les corps des victimes deviennent des engrenages vivants configure elle aussi une présence métonymique de l'Histoire, celle-ci étant le résultat de l'échec des Lumières, tournées en perversité.

Voilà donc, après cette prospection sur le texte de Révéroni, comment le traitement de la topique spatiale permet une lecture qui dépasse largement son apparente simplicité pour s'enrichir dans sa contextualisation historique, faisant du roman noir un genre fortement enraciné dans l'Histoire. D'un autre point de vue, celui de l'efficacité narrative, la machinerie spatiale déploie une grande versatilité : encadré dans une 
époque qui est essentiellement dynamisme, métamorphose et travestissement, l'espace est soumis à une manipulation constante, aboutissant à la construction d'une véritable mécanique du merveilleux. Or, la fascination du siècle par l'automatisme et la mécanique - et celle de Révéroni lui-même dont le regard porte l'empreinte de sa condition d'ingénieur militaire - se double en même temps d'une forte dose d'ironie, nouvel exemple de cette ambivalence constitutive que nous avons constaté tout au long de ces pages, et qui n'est autre que l'ambivalence même de l'Histoire dans ce tournant du siècle.

\section{NOTES}

1. Révéroni Saint-Cyr, Pauliska ou la perversité moderne (Paris: Desjonquères, 1991). Toutes les citations concernant le texte sont tirées de cette édition. Pour les préciser, il suffira donc d'indiquer les pages entre parenthèse.

2. Bien sûr, le concept d'utopie, tel qu'il a été défini par R. Trousson, n'est pas applicable à ce roman, où l'espace utopique n'existe pas. Par contre, nous pouvons observer - et cela à deux moments ponctuels dans le récit - l'apparition de certains éléments qui se situeraient dans la direction de la configuration d'un espace utopique, dans le sens d'une tendance vers la rêverie utopique: une microstructure sociale dépendante d'un sens de collectivisme, un système économique qui assure la survivance du groupe, la formation d'un certain esprit idéologique et une présence critique face à l'Histoire.

3. «J'étais si fatiguée que je préférais rester dans mes vêtements de paysanne, et c'était un spectacle assez piquant de voir la simple Petrowna, au milieu d'un cercle devenu très brillant, recueillir tous les hommages, toutes les attentions de la noblesse persécutée dans sa caste.» (page 41)

\section{AUTEUR}

\section{MARIA CONCEPCIÓN PÉREZ}

Université de Séville 